\title{
Entity-based Data Source Contextualization for Searching the Web of Data
}

\author{
Andreas Wagner ${ }^{\dagger}$, Peter Haase ${ }^{\ddagger}$, Achim Rettinger ${ }^{\dagger}$, and Holger Lamm ${ }^{\ddagger}$ \\ ${ }^{\dagger}$ Karlsruhe Institute of Technology and ${ }^{\ddagger}$ fluid Operations \\ \{a.wagner,rettinger\}@kit.edu, peter.haase@fluidops.com
}

\begin{abstract}
To allow search on the Web of data, systems have to combine data from multiple sources. However, to effectively fulfill user information needs, systems must be able to "look beyond" exactly matching data sources and offer information from additional/contextual sources (data source contextualization). For this, users should be involved in the source selection process - choosing which sources contribute to their search results. Previous work, however, solely aims at source contextualization for "Web tables", while relying on schema information and simple relational entities. Addressing these shortcomings, we exploit work from the field of data mining and show how to enable Web data source contextualization. Based on a real-world use case, we built a prototype contextualization engine, which we integrated in a system for searching the Web of data. We empirically validated the effectiveness of our approach - achieving performance gains of up to $29 \%$ over the state-of-the-art.
\end{abstract}

\section{Introduction}

The amount of RDF on the Web, such as Linked Data, RDFa and Microformats, is large and rapidly increasing. RDF data contains descriptions of entities, with each description being a set of triples: $\{\langle s, p, o\rangle\}$. A triple associates an entity (subject) $s$ with an object $o$ via a predicate $p$. A set of triples forms a data graph.

Querying of Distributed Data Sources. RDF is oftentimes highly distributed, with each data source comprising one or more RDF graphs, cf. Fig. 1.

Example. Catalogs like Eurostat ${ }^{1}$ or Worldbank ${ }^{2}$ provide finance data, e.g., about the gross domestic product (GDP), which is spread across many sources.

However, in order to provide the user with her desired information, a system has to combine such distributed data. Processing queries in such a manner requires knowledge about what source features which information. This problem is commonly known as source selection: a system chooses data sources relevant for a given query (fragment). Previous works selected sources via indexes, e.g., [9, 13, 18], link-traversal, e.g., [10,13], or by using source meta-data, e.g., [6].

Data Source Contextualization. Existing approaches for source selection aim solely at a mapping of queries/query fragments to sources with $e x$ actly matching data $[6,9,10,13]$. In particular, such works do not consider what sources are actually about and how they relate to each other.

Example. Consider a user searching for GDP rates in the EU. A traditional system may discover sources in Eurostat to comprise matching data. However, other sources offer contextual information concerning, e.g., the national debt.

\footnotetext{
${ }^{1}$ http://ec.europa.eu/eurostat/

${ }^{2}$ http://worldbank.org
} 
Src. 1. tec00001 (Eurostat).

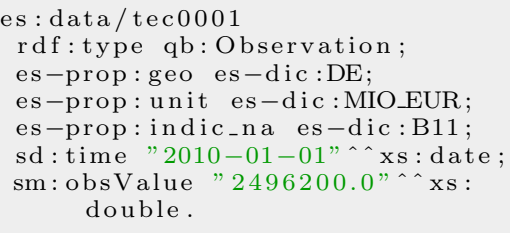

Src. 3. NY.GDP.MKTP.CN (Worldbank).

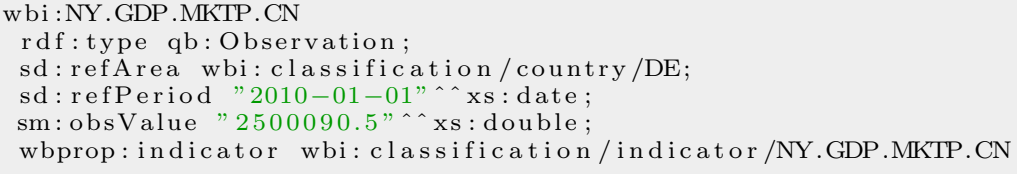

Fig. 1. Src. 1/3 describes Germany's GDP in 2010. They contextualize each other, as they feature varying observation values and properties for Germany's GDP. Src. 2 provides additional information, as it holds the German debt for 2010. Src. 1-3 each contain one entity: es:data/tec0001, es:data/gov_q_ggdebt, and wbi:NY.GDP.MKTP.CN. Every entity description equals the entire graph contained in its source.

Note, contextual sources are actually not relevant to the user's query, but relevant to her information need. Thus, integration of these "additional" sources provides a user with broader results in terms of result dimensions (schema complement) and result entities (entity complement). See our example in Fig. 1.

For enabling systems to identify and integrate sources for contextualization, we argue that user involvement during source selection is a key factor. That is, starting with an initial search result (obtained via, e.g., a SPARQL or keyword query), a user should be able to choose and change sources, which are used for result computation. In particular, users should be recommended contextual sources at each step of the search process. After modifying the selected sources, the results may be reevaluated and/or the query expanded.

Unfortunately, recent work on data source contextualization focuses on Web tables [2], while using top-level schema such as Freebase. Further, the authors restrict data to a simple relational form. We argue that such a solution is not a good fit for the schemaless, heterogeneous Web of data.

Contributions. This paper continues our work in [20]. More specifically, we provide the following contributions: (1) Previous work on Web table contextualization suffers from inherent drawbacks. Most notably, it is restricted to fixed-structured relational data and requires external schemata to provide additional information. Omitting these shortcomings, we present an entity-based solution for data source contextualization in the Web of data. Our approach is based on well-known data mining strategies and does not require schema information or data adhering to a particular form. (2) We implemented our system, the data-portal, based on a real-world use case, thereby showing its practical relevance and feasibility. A prototype version of this portal is publicly available and is currently tested by a pilot customer. ${ }^{3}$ (3) We conducted two user studies to empirically validate the effectiveness of the proposed approach: our system outperforms the state-of-the-art with up to $29 \%$.

\footnotetext{
3 http://data.fluidops.net/
} 
Outline. In Sect. 2, we present our use case. We give preliminaries in Sect. 3, outline the approach in Sect. 4, and present its implementation in Sect. 5. We discuss the evaluation in Sect. 6, related work in Sect. 7, and conclude in Sect. 8.

\section{Use Case Scenario}

In this section, we introduce a real-world use case to illustrate challenges w.r.t. data source selection during searching the Web of data. The scenario is provided by a pilot user in the financial industry and available online. ${ }^{4}$

In their daily work, financial researchers heavily rely on a variety of open and closed Web data sources, in order to provide prognoses of future trends. A typical example is the analysis of government debt. During the financial crisis in 2008-2009, most European countries made high debts. To lower doubts about repaying these debts, most countries set up a plan to reduce their public budget deficits. To analyze such plans, a financial researcher requires an overview of public revenue and expenditure in relation to the gross domestic product (GDP). To measure this, she needs information about the deficit target, the revenue/expenditure/deficit, and GDP estimates. This information is publicly available, provided by catalogs like Eurostat and Worldbank. However, it is spread across a huge space of sources. That is, there is no single source satisfying her information needs - instead data from multiple sources has to be identified and combined. To start her search process, a researcher may give "gross domestic product" as keyword query. The result is GDP data from a large number of sources. At this point, data source selection is "hidden" from the researcher, i.e., sources are solely ranked via number and quality of keyword hits. However, knowing where her information comes from is critical. In particular, she may want to restrict and/or know the following meta-data:

- General information about the data source, e.g., the name of the author and a short description of the data source contents.

- Information about entities contained in the data source, e.g., the single countries of the European Union.

- Description about the dimensions of the observations, e.g., the covered time range or the data unit of the observations.

By means of faceted search, the researcher finally restricts her data source to tec00001 (Eurostat, Fig. 1) featuring "Gross domestic product at market prices". However, searching the data source space in such a manner requires extensive knowledge. Further, the researcher was not only interested in plain GDP data - she was also looking for additional information.

For this, a system should suggest data sources that might be of interest, based on sources known to be relevant. These contextual sources may feature related, additional information w.r.t. current search results/sources. For instance, data sources containing information about the GDP of further countries or with a different temporal range. This way, the researcher may discover new sources more easily, as one source of interest links to another - allowing her to explore the space of sources.

\footnotetext{
${ }^{4}$ http://data.fluidops.net/resource/Demo_GDP_Germany
} 


\section{Preliminaries}

Data Model. Data in this work is represented as RDF:

Definition 1 (RDF Triple, RDF Graph). Given a set of URIs $\mathcal{U}$, blank nodes $\mathcal{B}$, and a set of literals $\mathcal{L}, t=\langle s, p, o\rangle \in \mathcal{U} \cup \mathcal{B} \times \mathcal{U} \times(\mathcal{U} \cup \mathcal{L} \cup \mathcal{B})$ is a $\mathrm{RDF}$ triple. $A$ RDF graph $\mathcal{G}=(\mathcal{V}, \mathcal{E})$ is defined by vertices $\mathcal{V}=\mathcal{U} \cup \mathcal{L} \cup \mathcal{B}$ and a set of triples as edges $\mathcal{E}=\{\langle s, p, o\rangle\}$.

A data source may contain one or more RDF graphs:

Definition 2 (RDF Data Source). A data source $D_{i} \in \mathcal{D}$ is a set of $n R D F$ graphs, i.e., $D_{i}=\left\{\mathcal{G}_{1}^{i}, \ldots, \mathcal{G}_{n}^{i}\right\}$, with $\mathcal{D}$ as set of all sources.

Notice, the above definition abstracts from the data access, e.g., via HTTP GET requests. In particular, Def. 2 also covers Linked Data sources, see Fig. 1.

Entity Model. Given a data source $D_{i}$, an entity $e$ is a subject that is identified with a URI $d_{e}$ in $\mathcal{G}_{j}^{i}$. Entity $e$ is described via a connected subgraph of $\mathcal{G}_{j}^{i}$ containing $d_{e}$ (called $\mathcal{G}_{e}$ ). Subgraphs $\mathcal{G}_{e}$, however, may be defined in different ways [7]. For our work, we used the concise bound description, where all triples with subject $d_{e}$ are comprised in $\mathcal{G}_{e}$. Further, if an object is a blank node, all triples with that blank node as subject are also included and so on [7].

Example. We have 3 subjects in Fig. 1 and each of them stands for an entity. Every entity description, $\mathcal{G}_{e}$, is a one-hop graph. For instance, the description for entity es:data/tec0001 comprises all triples in Src. 1.

Kernel Functions. We compare different entities by comparing their descriptions, $\mathcal{G}_{e}$. For this, we make use of kernel functions [19]:

Definition 3 (Kernel function). Let $\kappa: \mathcal{X} \times \mathcal{X} \mapsto \mathbb{R}$ denote a kernel function such that $\kappa\left(x_{1}, x_{2}\right)=\left\langle\varphi\left(x_{1}\right), \varphi\left(x_{2}\right)\right\rangle$, where $\varphi: \mathcal{X} \mapsto \mathcal{H}$ projects a data space $\mathcal{X}$ to a feature space $\mathcal{H}$ and $\langle\cdot, \cdot\rangle$ refers to the scalar product.

Note, $\varphi$ is not restricted, i.e., a kernel is constructed without prior knowledge about $\varphi$. We will give suitable kernels for entity descriptions, $\mathcal{G}_{e}$, in Sect. 4 .

Clustering. We use $k$-means [11] as a simple and scalable algorithm for discovering clusters, $C_{i}$, of entities in data sources $\mathcal{D}$. It works as follows [11]:

(1) Choose $k$ initial cluster centers, $m_{i}$. (2) Based on a dissimilarity function, dis, an indicator function is given as: $\mathbb{1}\left(e, C_{i}\right)$ is 1 if $\operatorname{dis}\left(e, C_{i}\right)<\operatorname{dis}\left(e, C_{j}\right), \forall j \neq i$ and 0 otherwise. That is, $\mathbb{1}\left(e, C_{i}\right)$ assigns each entity $e$ to its "closest" cluster $C_{i}$. (3) Update cluster centers $m_{i}$ and reassign, if necessary, entities to new clusters. (4) Stop if convergence threshold is reached, e.g., no (or minimal) reassignments occurred. Otherwise go back to (2). A key problem is defining a dissimilarity function for entities in $\mathcal{D}$. Using kernels we may define such a function - as we will show later, cf. Sect. 4.1.

Problem. We address the problem of finding contextual data sources for a given source. Contextual sources should: (a) Add new entities that refer to the same real world object as given ones (entity complement). (b) Add entities with same URI identifier, but have new properties in their description (schema complement). Note, (a) and (b) are not disjoint, i.e., some entities may be new and add additional properties. 
Example. In Fig. 1 Src. 3 contextualizes Src. 1 in terms of both, entity as well as schema complement. That is, Src. 3 adds entity wbi:NY. GDP.MKTP.CN, while providing new properties and a different GDP value. In fact, even Src. 2 contextualizes Src. 1, as entities in both sources refer to the real world object "Germany" - one source captures the GDP, while the other describes the debt.

\section{Entity-based Data Source Contextualisation}

Approach. The intuition behind our approach is simple: if data sources contain similar entities, they are somehow related. In other words, we rely on (clusters of) entities to capture the "latent" semantics of data sources.

More precisely, we start by extracting entities from given data sources. In a second step, we apply clustering techniques, to mine for entity groups. Notice, for the $k$-means clustering we employ a kernel function as similarity measure. This way, we abstract from the actual entity representation, i.e., RDF graphs, and use a high-dimensional space (feature space) to increase data comparability/separability [19]. Last, we rely on entity clusters for relating data sources to each other and compute a contextualization score based on these clusters. Note, only the last step is done online - all other steps/computations are offline.

Discussion. We argue our approach to allow for some key advantages: (1) We decouple representation of source content and source similarity, by relying on entities for capturing the overall source semantics. (2) Our solution is highly flexible as it allows to "plug-in" application-specific entity definitions/extraction strategies, entity similarity measures, and contextualization heuristics. That is, we solely require an entity to be described as a subgraph, $\mathcal{G}_{e}$, contained in its data source $D$. Further, similarity measures may be based on any valid kernel function. Last, various heuristics proposed in [2] could be adapted for our approach. (3) Exploiting clusters of entities allows for a scalable and maintainable approach, as we will outline in the following.

\subsection{Related Entities}

In order to compare data sources, we first compare their entities with each other. That is, we extract entities, measure similarity between them and finally cluster them (Fig. 2). All of these procedures are offline.

Entity Similarity. We start by extracting entities from each source $D_{i} \in \mathcal{D}$ : First, for scalability reasons, we go over all subjects in RDF graphs in $D_{i}$ and collect an entity sample, with every entity $e$ having the same probability of being selected. Then, we crawl the concise bound description [7] for each entity $e$ in the sample. For cleaning $\mathcal{G}_{e}$, we apply standard data cleansing strategies to fix, e.g., missing or wrong data types. Having extracted entities, we define a similarity measure relating pairs of entities. For this, we use two kinds of kernels: (1) kernels for structural similarities $\kappa^{s}$ and (2) those for literal similarities $\kappa^{l}$.

With regard to the former, we measure structural "overlaps" between entity descriptions, $\mathcal{G}_{e}^{\prime}$ and $\mathcal{G}_{e}^{\prime \prime}$, using graph intersections [16]:

Definition 4 (Graph Intersection $\left.\mathcal{G}^{\prime} \cap \mathcal{G}^{\prime \prime}\right)$. Let the intersection between two graphs, denoted as $\overline{\mathcal{G}}=\mathcal{G}^{\prime} \cap \mathcal{G}^{\prime \prime}$, be given by $\overline{\mathcal{V}}=\mathcal{V}^{\prime} \cap \mathcal{V}^{\prime \prime}$ and $\overline{\mathcal{E}}=\mathcal{E}^{\prime} \cap \mathcal{E}^{\prime \prime}$. 
(a) Extracted Entities

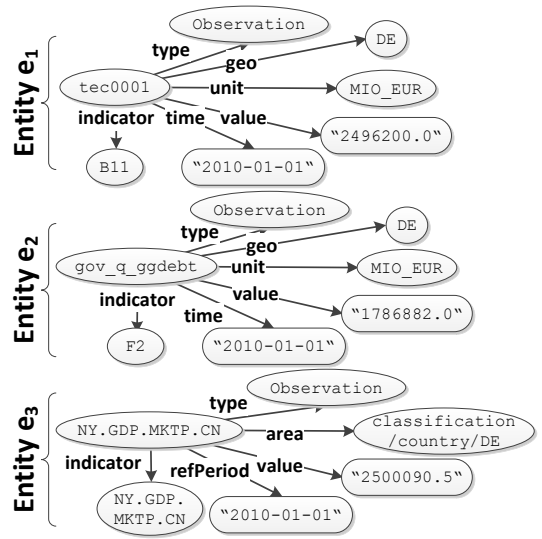

(b) Entity Similarity

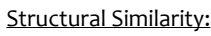

Intersection Graphs

$\mathrm{G}_{\mathrm{e} 1} \cap \mathrm{G}_{\mathrm{e} 2}$

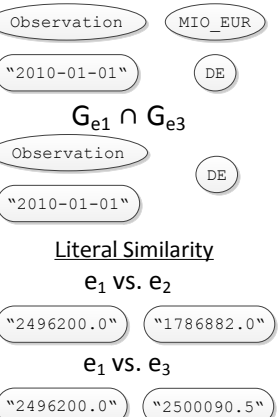

(c) Entity Clusters

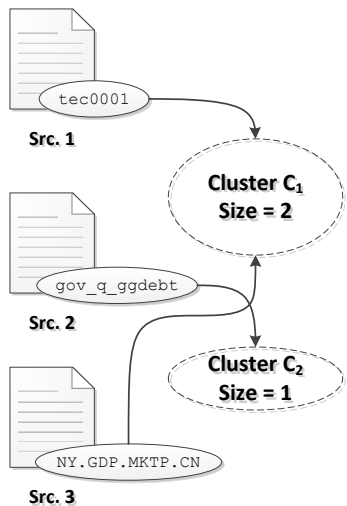

Fig. 2. (a) Extracted entities $e_{1}, e_{2}$ and $e_{3}$ from Fig. 1. (b) Structural similarities as intersection graphs $\mathcal{G}_{e 1} \cap \mathcal{G}_{e 2}$ and $\mathcal{G}_{e 1} \cap \mathcal{G}_{e 3}$. Literal similarities for entity $e_{1}$ vs. $e_{2}$ and $e_{1}$ vs. $e_{3}$. Note, exact matching literals are omitted, as they are covered already by the intersection graphs. Comparison of $e_{2}$ vs. $e_{3}$ is omitted due to space reasons.

(c) Entities grouped in $k=2$ clusters.

We aim at connected structures in $\mathcal{G}_{e}^{\prime} \cap \mathcal{G}_{e}^{\prime \prime}$. Thus, we define a path [16]:

Definition 5 (Path). Let a path in a graph $\mathcal{G}$ be defined as a sequence of vertices and triples $v_{1},\left\langle v_{1}, p_{1}, v_{2}\right\rangle, v_{2}, \ldots, v_{n}$, with $\left\langle v_{i}, p_{i}, v_{i+1}\right\rangle \in \mathcal{E}$, having no cycles. The path length is given by the number of contained triples. The set of all paths, up to length $l$, in $\mathcal{G}$ is denoted as path $(\mathcal{G})$.

The corresponding path kernel is [16]:

Definition 6 (Path Kernel $\left.\kappa^{s}\right)$. A path kernel is $\kappa_{l, \lambda}^{s}\left(\mathcal{G}_{1}, \mathcal{G}_{2}\right)=\sum_{i=1}^{l} \lambda^{i} \mid\{p \mid$ $p \in$ paths $\left._{i}\left(\mathcal{G}_{1} \cap \mathcal{G}_{2}\right)\right\} \mid$, with $\lambda>0$ as discount factor for path length.

Note, [16] introduced further kernels, however, we found path kernels to be simple and perform well in our experiments, cf. Sect. 6 .

Example. Extracted entities from sources in Fig. 1 are given in Fig. 2-a. In Fig. 2-b, we compare the structure of tec0001 (short: $e_{1}$ ) and gov_q_ggdebt (short: $e_{2}$ ). For this, we compute an intersection: $\mathcal{G}_{e 1} \cap \mathcal{G}_{e 2}$. This yields a set of 4 paths, each with length 0 . The unnormalized kernel value is $\lambda^{0} \cdot 4$.

For literal similarities, one can use different kernels $\kappa^{l}$ on, e.g., strings or numbers [19]. For space reasons, we restrict presentation to the string subsequence kernel, $\kappa_{s}^{l}$ [15]. A numerical kernel, $\kappa_{n}^{l}$, is outlined in our extended report [21].

Definition 7 (String Subsequence Kernel $\kappa_{s}^{l}$ ). Let $\Sigma$ denote a vocabulary for strings, with each string $s$ as finite sequence of characters in $\Sigma$. Let $s[i$ : $j$ ] denote a substring $s_{i}, \ldots, s_{j}$ of $s$. Further, let $u$ be a subsequence of $s$, if indices $i=\left(i_{1}, \ldots, i_{|u|}\right)$ exist with $1 \leq i_{1} \leq i_{|u|} \leq|s|$ such that $u=s[i]$. The length $l(i)$ of subsequence $u$ is $i_{|u|}-i_{1}+1$. Then, a kernel function $\kappa^{l} i s$ defined as sum over all common, weighted subsequences for strings $s, t: \kappa_{\lambda}^{l}(s, t)=$ $\sum_{u} \sum_{i: u=s[i]} \sum_{j: u=t[j]} \lambda^{l}(i) \lambda^{l}(j)$, with $\lambda$ as decay factor. 
Example. For instance, strings "MI" and "MIO_EUR" share a common subsequence "MI" with $i=(1,2)$. Thus, the unnormalized kernel is $\lambda^{2}+\lambda^{2}$.

As literal kernels, $\kappa^{l}$, are only defined for two literals, we sample over every possible literal pair (with the same data type) for two given entities and aggregate the needed kernels for each pair. Finally, we aggregate structure kernel, $\kappa^{s}$ and literal kernels, $\kappa^{l}$, resulting in one single kernel [19]:

$$
\kappa\left(e^{\prime}, e^{\prime \prime}\right)=\kappa^{s}\left(\mathcal{G}_{e^{\prime}}, \mathcal{G}_{e^{\prime \prime}}\right) \bigoplus_{o_{1}, o_{2} \in \operatorname{sample}\left(\mathcal{G}_{e^{\prime}}, \mathcal{G}_{e^{\prime \prime}}\right)} \kappa^{l}\left(o_{1}, o_{2}\right)
$$

We use a weighted summation as aggregation $\oplus$ (we obtained weights experimentally). The dissimilarity between $e^{\prime}$ and $e^{\prime \prime}$ is given as Euclidean distance [22]:

$$
\operatorname{dis}^{2}\left(e^{\prime}, e^{\prime \prime}\right):=\left\|\varphi\left(e^{\prime}\right)-\varphi\left(e^{\prime \prime}\right)\right\|^{2}=\kappa\left(e^{\prime}, e^{\prime}\right)-2 \kappa\left(e^{\prime}, e^{\prime \prime}\right)+\kappa\left(e^{\prime \prime}, e^{\prime \prime}\right)
$$

Entity Clustering. Using this dissimilarity measure, we may learn clusters of entities. Notice, our algorithm does not separate the input data (graphs $\mathcal{G}_{e}$ ), but instead its representation in the feature space. Based on [22], cluster center $m_{i}$ in the feature space is: $m_{i}=\frac{1}{\left|C_{i}\right|} \sum \mathbb{1}\left(\varphi(e), C_{i}\right) \varphi(e)$. Distance between a projected entity $\varphi(e)$ and $m_{i}$ is given by [22]:

$$
\begin{aligned}
\operatorname{dis}^{2}\left(\varphi(e), m_{i}\right) & =\left\|\varphi(e)-m_{i}\right\|^{2}=\kappa(e, e)+f\left(e, C_{i}\right)+g\left(C_{i}\right), \text { with } \\
f\left(e, C_{i}\right) & :=-\frac{2}{\left|C_{i}\right|} \sum_{j} \mathbb{1}\left(\varphi\left(e_{j}\right), C_{i}\right) \kappa\left(e, e_{j}\right) \\
g\left(C_{i}\right) & :=\frac{1}{\left|C_{i}\right|^{2}} \sum_{j} \sum_{l} \mathbb{1}\left(\varphi\left(e_{j}\right), C_{i}\right) \mathbb{1}\left(\varphi\left(e_{l}\right), C_{i}\right) \kappa\left(e_{j}, e_{l}\right)
\end{aligned}
$$

Now, $k$-means can be applied as introduced in Sect. 3 .

Example. In Fig. 2-c, we found two entity clusters. Here, structural similarity was higher for entities $e_{1}$ vs. $e_{2}$ than for $e_{1}$ vs. $e_{3}$. However, numerical similarity between $e_{1}$ vs. $e_{3}$ was stronger: "2496200.0" was closer to "2500090.5" as "1786882". As we weighted literal similarity to be more important than structural similarity, this leads to $e_{1}$ and $e_{3}$ forming one cluster. In fact, such a clustering is intuitive, as $e_{1}$ and $e_{3}$ is about GDP, while $e_{2}$ is concerned with debt.

\subsection{Related Data Sources}

Given a data source $D^{\prime}$, we score its contextualization w.r.t. another source $D^{\prime \prime}$, using entities contained in $D^{\prime}$ and $D^{\prime \prime}$. Note, in contrast to previous work [2], we do not rely on any kind of "external" information, such as top-level schema. Instead, we solely exploit semantics as captured by entities.

Contextualisation Score. Similar to [2], we compute two scores, ec $\left(D^{\prime \prime} \mid\right.$ $\left.D^{\prime}\right)$ and $\operatorname{sc}\left(D^{\prime \prime} \mid D^{\prime}\right)$, for a data source $D^{\prime \prime}$, given another source $D^{\prime}$. The former is an indicator for the entity complement of $D^{\prime \prime}$ w.r.t. $D^{\prime}$. That is, how many new, similar entities does $D^{\prime \prime}$ contribute to given entities in $D^{\prime}$. The latter score judges how many new "dimensions" are added by $D^{\prime \prime}$, to those present in $D^{\prime}$ (schema complement). Both scores are aggregated to a contextualization score for data source $D^{\prime \prime}$ given $D^{\prime}$. 
Let us first define an entity complement score $e c: \mathcal{D} \times \mathcal{D} \mapsto[0,1]$. We may measure $e c$ simply by counting the overlapping clusters between both sources:

$$
e c\left(D^{\prime \prime} \mid D^{\prime}\right):=\sum_{C_{j} \in \operatorname{cluster}\left(D^{\prime}\right)} \frac{\mathbb{1}\left(C_{j}, D^{\prime \prime}\right)\left|C_{j}\right|}{\left|C_{j}\right|}
$$

with cluster as function mapping data sources to clusters their entities are assigned to. Further, let $\mathbb{1}(C, D)$ by an indicator function, returning 1 if cluster $C$ is associated with data source $D$ via one or more entities.

Considering the schema complement score $s c: \mathcal{D} \times \mathcal{D} \mapsto[0,1]$, we aim to add new dimensions (properties) to those already present. Thus, $s c$ is given as:

$$
\operatorname{sc}\left(D^{\prime \prime} \mid D^{\prime}\right):=\sum_{C_{j} \in \operatorname{cluster}\left(D^{\prime \prime}\right)} \frac{\left|\operatorname{props}\left(C_{j}\right) \backslash \bigcup_{C_{i} \in \operatorname{cluster}\left(D^{\prime}\right)} \operatorname{props}\left(C_{i}\right)\right|}{\left|\operatorname{props}\left(C_{j}\right)\right|}
$$

with props as function that projects a cluster $C$ to a set of properties, where each property is contained in a description of an entity in $C$.

Finally, a contextualization score $c s$ is obtained by a monotonic aggregation of $e c$ and $s c$. In our case, we apply a simple, weighted summation:

$$
\operatorname{cs}\left(D^{\prime \prime} \mid D^{\prime}\right):=1 / 2 \cdot \operatorname{ec}\left(D^{\prime \prime} \mid D^{\prime}\right)+1 / 2 \cdot \operatorname{sc}\left(D^{\prime \prime} \mid D^{\prime}\right)
$$

Example. Assuming Src. 1 is given, $\operatorname{cs}\left(D_{2} \mid D_{1}\right)=\operatorname{cs}\left(D_{3} \mid D_{1}\right)=1 / 2$, because $e c\left(D_{3} \mid D_{1}\right)=1$ and $\operatorname{sc}\left(D_{3} \mid D_{1}\right)=0$ (the other way around for $\left.D_{2} \mid D_{1}\right)$. These scores are meaningful, because $D_{2}$ adds additional properties (debt), while $D_{3}$ complements $D_{1}$ with entities (GDP). See also Fig. 2-c.

Runtime Behavior and Scalability. Regarding online performance, i.e., computation of contextualization score cs, given the offline learned clusters, we aimed at simple and lightweight heuristics. For ec only an assignment of data sources to clusters (function cluster()) and cluster size $|C|$ is needed. Further, measure $s c$ only requires an additional mapping of clusters to "contained" properties (function props () ). All necessary statistics are easily kept in memory.

With regard to the offline clustering behavior, we expect our approach to perform well, as existing work on kernel $k$-means clustering showed such approaches to scale to large data sets $[1,5,22]$.

\section{Searching the Web of Data via Source Contextualization}

Based on our real-world use case (Sect. 2), we show how a source contextualization engine may be integrated in a real-world query processing system for the Web of data. Notice, an extended system description is included in our report [21].

Overview. Towards an active involvement of users in the source selection process, we implemented a data portal, which offers a data source space exploration as well as a distributed query processing service.

Using the former, users may explore the space of sources, i.e., search and discover data sources of interest. Here, the contextualization engine fosters discovery of relevant sources during exploration. The query processing service, on the other hand, allows queries to be federated over multiple sources. 


\section{FluidOps Data Portal}

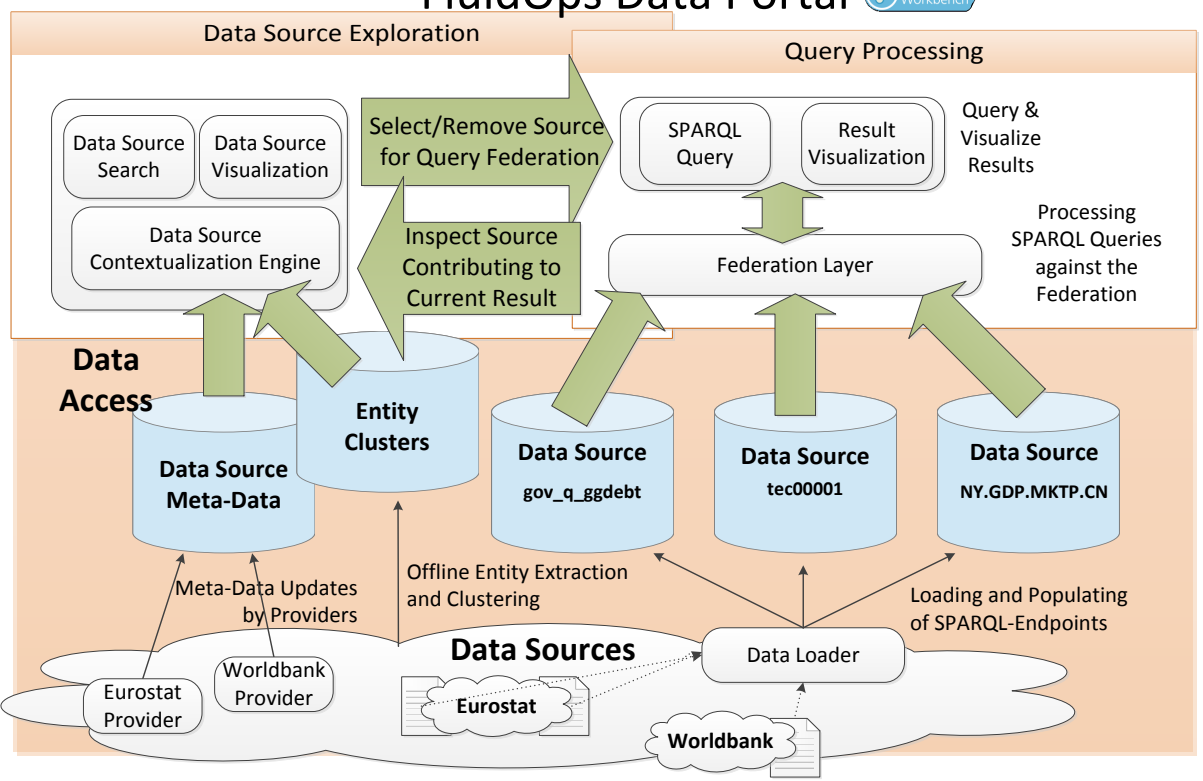

Fig. 3. Our data portal offers two services: source space exploration and query processing. The source contextualization engine is integrated as a key component of the source space exploration. For this, data source meta-data is loaded and entities are extracted/clustered. For query processing, each data source is mapped to a SPARQL endpoint, from which data is accessed via a data-loader.

Interaction between both services is tight and user-driven. In particular, sources discovered during source exploration may be used for answering queries. On the other hand, sources employed for result computation may be inspected and other relevant sources may be found via contextualization.

The data portal is based on the Information Workbench [8] and a running prototype is available. ${ }^{5}$ Following our use-case (Sect. 2), we populated the system with statistical data sources from Eurostat and Worldbank. This population involved an extraction of meta-data, which is used to load the sources locally as well as give users insights into the source. Every data source is stored in a triple store - accessible via a SPARQL endpoint. See Fig. 3 for an overview.

Source Exploration and Selection. A typical search process starts with looking for "the right" sources. That is, a user begins with exploration of the data source space. For instance, she may issue a keyword query "gross domestic product", yielding sources with matching words in their meta-data. If this query does not lead to sources suitable for her information need, a faceted search interface or a tag-cloud may be used. For instance, she refines her sources via entity "Germany" in a faceted search, Fig.4-a. Once the user discovered a source of interest, its structure as well as entity information is shown. For example, a source description for GDP (current US\$) is given in Fig.4-b/c. Note, entities used here have been extracted by our approach and are visualized by means of a map. Using

\footnotetext{
${ }^{5}$ http://data.fluidops.net/
} 

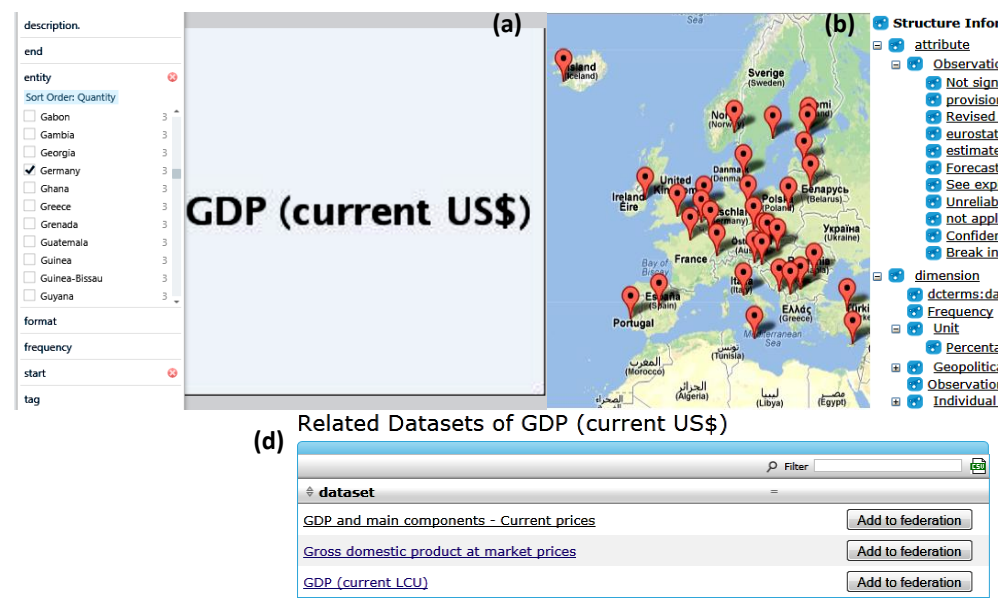

Fig. 4. (a) Faceted search exploration through sources. (b) $+(c)$ Source information for GDP (current US\$) based on its entities and schema. (d) Contextualization sources for GDP (current US\$).

these rich source descriptions, a user can get to know the data and data sources before issuing queries. Further, for every source a ranked list of contextualization sources is given. For GDP (current US\$), e.g., source GDP at Market Prices is recommended, Fig.4-d. This way, the user is guided from one source of interest to another. At any point, she may select a particular source for querying. Eventually, she not only knows her relevant sources, but has also gained first insights into data schema and entities.

Processing Queries over Selected Sources. Say, a user has chosen GDP (current US\$) as well as its contextualization source GDP at Market Prices (Fig. 4-d). Due to her previous exploration, she knows that the former provides the German GDP from 2000 - 2010, while the second source features GDP from years 2011 and 2012. Thus, she may issue a SPARQL query over both sources to visualize the GDP over the years 2000 - 2012, cf. Fig. 5.

\section{Evaluation}

We now discuss evaluation results to analyze the effectiveness of our approach. We conducted two experiments: (1) Effectiveness w.r.t. a gold standard, thereby measuring the accuracy of the contextualization score. (2) Effectiveness w.r.t. data source search result augmentation. In other words, we ask: How useful are top-ranked contextualization sources in terms of additional information?

Participants. Experiment (1) and (2) were performed by two different groups, each comprising 14 users. Most participants had an IT background and/or were CS students. Three users came from the finance sector. The users vary in age, 22 to 37 years and gender. All experiments were unsupervised.

Data Sources and Clustering. Following our use case in Sect. 2, we employed Linked Data sources from Eurostat ${ }^{6}$ and Worldbank ${ }^{7}$. Both provide a

\footnotetext{
${ }^{6}$ http://eurostat.linked-statistics.org/

${ }^{7}$ http://worldbank.270a.info
} 


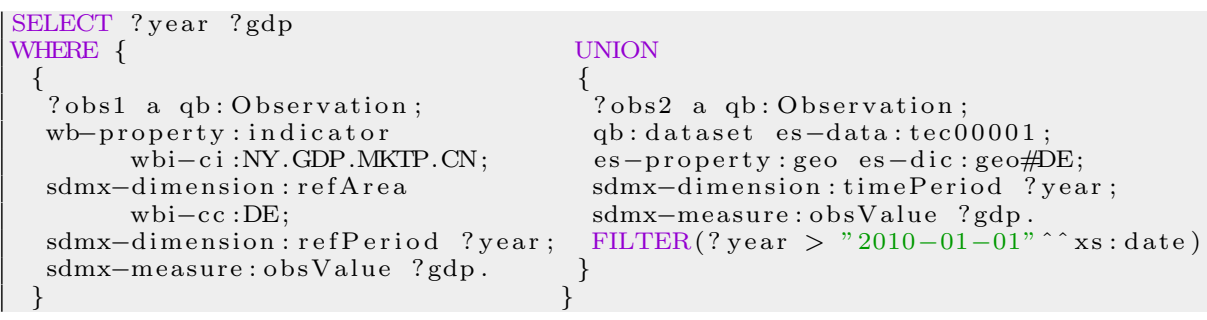

Course of Germany's GDP, in millions of Euro

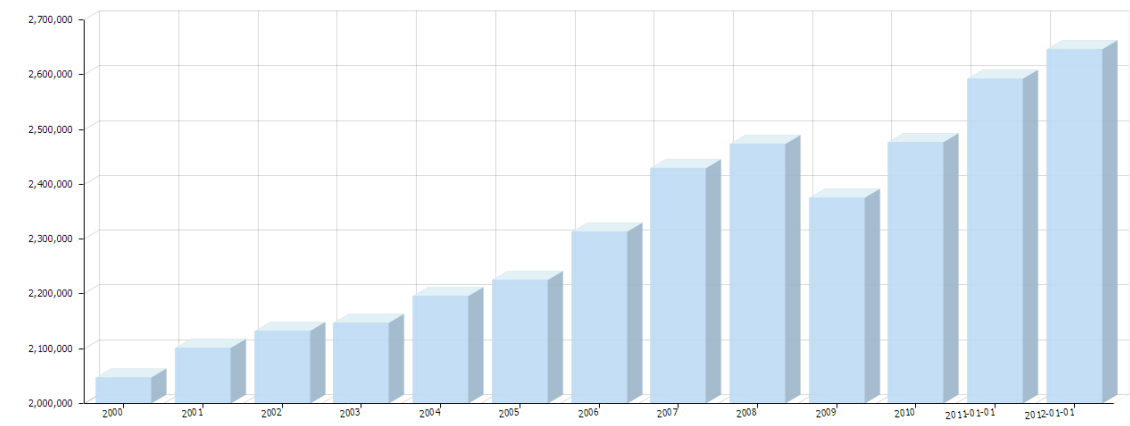

Fig. 5. Query and result visualization for Germany's GDP from 2000-2012. Data sources were selected during source exploration, see Fig. 4.

large set of data sources comprising statistical information: Eurostat holds $5 \mathrm{~K}$ sources (8000 $M$ triples), while Worldbank has $76 K$ sources (167 $M$ triples). Note, for Eurostat we simply used instances of void:Dataset as sources and for Worldbank we considered available dumps as sources. Further, sources varied strongly in their size and \# entities. While small sources contained $\leq 10$ entities, large sources featured $\geq 1 K$ entities. For extracting/sampling of entities, we restricted attention to instances of qb:Observation. We employed the $k$-means algorithm with $k=30 K$ (chosen based on experiments with different values for $k$ ) and initiated the cluster centers via random entities.

Queries. Based on evaluation queries in [2], domain experts constructed 15 queries. A query listing is depicted in Table 1. This query load was designed to be "broad" w.r.t. the following dimensions: (1) We cover a wide range of topics, which may be answered by Eurostat and Worldbank. (2) "Best" results for each query are achieved by using multiple sources from both datasets. (3) \# Sources and \# entities relevant for a particular query vary.

Systems. We implemented entity-based source contextualization (EC) as described in Sect. 4. In particular, we made use of three kernels for capturing entity similarity: a path kernel, a substring kernel, and a numerical kernel $[16,19]$. As baselines we used two approaches: a schema-based contextualization SC [2] and a keyword-based contextualization KC. SC maps entities in data sources to toplevel schemata and finds complementary sources based on schema as well as label similarities of mapped entities. More precisely, the baseline is split in two approaches: $\mathbf{S C}_{e c}$ and $\mathbf{S C}_{s c}$. $\mathbf{S C}_{e c}$ aims at entity complements, i.e., data sources with similar schema, but different entities. $\mathbf{S C}_{s c}$ targets data sources having complementary schema, but holding the same entities. Last, the KC approach treats 


\begin{tabular}{l|ccc|c|ccc} 
& Keywords & \#Entities & \#Sources & & \multicolumn{2}{c}{ Keywords } & \#Entities \\
\hline Q1 & country debt & 3,155 & 1,981 & Q9 & international trade & 2,444 & 1,085 \\
Q2 & country gdp & 4,265 & 1,969 & Q10 & national trade & 2,971 & 1,199 \\
Q3 & country population & 4,076 & 3,868 & Q11 & price indices & 10,490 & 4930 \\
Q4 & energy consumption & 5,194 & 651 & Q12 & prison population & 5,135 & 629 \\
Q5 & fish species & 2,323 & 157 & Q13 & research expenditure & 5,287 & 201 \\
Q6 & fish areas & 6,664 & 316 & Q14 & school leavers & 2,722 & 637 \\
Q7 & forest area & 3,141 & 707 & Q15 & unemployment rate & 2,914 & 266 \\
Q8 & gas emissions & 5,470 & 722 & $\sum$ & & 66,251 & 19,318
\end{tabular}

Table 1. Queries with their number of relevant entities and sources.

every data source as a bag-of-words and judges the relevance of a source w.r.t. a query by the number and quality of its matching keywords.

\subsection{Effectiveness of Contextualisation Score}

Gold Standard. We calculated a simple gold standard that ranks pairs of sources based on their contextualization. That is, for each query in Table 1, we randomly selected one "given" source and 5 "additional" sources - all of which contained the query keywords. Then, 14 users were presented that given source and had to rank how well each of the 5 additional sources contextualizes it (scale on 0 - 5, where higher is better). To judge the sources' contents, users were given a schema description and a short source extract. We aggregated the user rankings, thereby obtaining a gold standard ranking.

Metric. We applied the footrule distance [12] as rank-distance indicator, which measures the accuracy of the evaluation systems vs. gold standard. Footrule distance is: $\frac{1}{k} \sum_{i=1, \ldots, k}\left|\operatorname{rank}_{a}(i)-\operatorname{rank}(i)\right|$, with $\operatorname{rank}_{a}(i)$ and $\operatorname{rank}(i)$ as approximated and gold standard rank for the "additional" source $D_{i}$.

Results. Fig. 6-a/d give an overview over results of EC and SC. Note, we excluded the KC baseline, because it was too simplistic. That is, reducing sources to bags-of-words, one may only intersect these bags for two given sources. This yielded, however, no meaningful contextualization scores/ranking.

We noticed EC to lead to a good and stable performance over all queries (Fig. 6-a) as well as data source sizes (Fig. 6-d). Overall, EC could outperform $\mathrm{SC}_{s c}$ by $5.7 \%$ and $\mathrm{SC}_{e c}$ by $29 \%$. Further, as shown in Fig. 6-d, while we observed a decrease in rank distance for all systems in source and entity sample size, EC yielded the best results. We explain these results with our fine-grained source semantics captured by entity clusters. Note, most of our employed contextualization heuristics are very similar to those from SC. However, we observed SC performance to strongly vary with the quality of its schema mappings. Given an accurate classification of entities contained in a particular source, SC was able to effectively relate that source with others. However, if entities were mapped to "wrong" concepts, it greatly affected computed scores. In contrast, our approach relied on clusters learned from instance data, thereby achieving a "more reliable" mapping from sources to their semantics (clusters).

On the other hand, we observed EC to result in equal or, for 2 outlier queries with many "small" sources, worse performance than $\mathrm{SC}_{s c}$ (cf. Fig. 6-d). In particular, given query $\mathrm{Q} 2, \mathrm{SC}_{s c}$ could achieve a better ranking distance by $20 \%$. We explain such problematic queries with our simplistic entity sampling. Given 


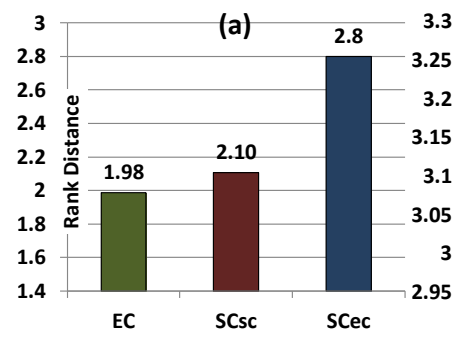

(d)

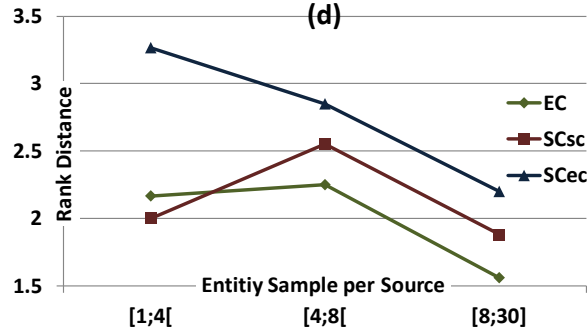

$[4 ; 8[$
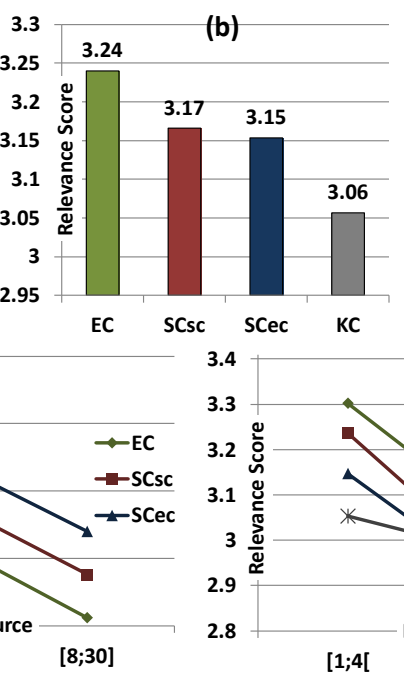

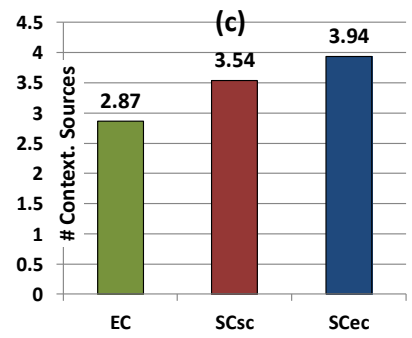

(e)

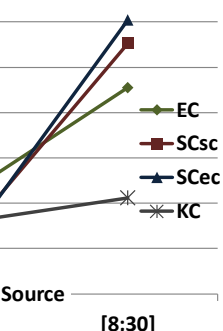

Fig. 6. (a) Exp-1: Rank distance as average over all queries. (b) Exp-2: Relevance score per source, as average over all queries. (c) Exp-2: \# Sources for contextualization per source, as average over all queries. (d) Exp-1: Rank distance average over all queries vs. average \# sampled entities per source. (e) Exp-2: Relevance score as average over all queries vs. average \# sampled entities per source.

small sources, only few entities were included in a sample, which unfortunately pointed to "misleading" clusters. Note, we currently only use a uniform random sample for selecting entities from sources. We expect better results with more refined techniques for discovering important entities for a particular source.

Last, we observed $\mathbf{S C}_{e c}$ to lead to much less accurate rankings as $\mathbf{S C}_{s c}$ and EC. This is due to exact matching of entity labels: $\mathbf{S C}_{e c}$ did not capture common substrings (as EC did), but solely relied on a boolean similarity matching.

\subsection{Effectiveness of Augmented Data Source Results}

Metric. Following [2], we employ a reordering of data source search results as metric: (1) We obtain top 100 data sources via the KC baseline for every query. Here, the order is determined solely by number and quality of keyword hits in the sources' bags-of-words. (2) Via SC and EC we reorder the first 10 results:

For each data source $D_{i}$ in the top-10 results, we search the top-100 sources for a $D_{j}$ that contextualizes $D_{i}$ best. If we find a $D_{j}$ with contextualization score higher than a threshold, we move $D_{j}$ after $D_{i}$ in the top-10 results.

This reordering procedure yields three different top-10 source rankings: one via KC and two (reordered ones) from SC/EC. For every top-10 ranking, users provided a relevance feedback for each source w.r.t. a given query, using a scale 0 - 5 (higher means "more relevant"). For this, users had a schema description and short source extract for every source. Last, we aggregated these relevancy judgments for each top-10 ranking and query.

Results. User relevance scores are depicted in Fig. 6-b/e. Overall, EC yielded an improved ranking, i.e., it ranked more relevant sources w.r.t. a given query. More precisely, EC outperforms $\mathrm{SC}_{s c}$ with $2.5 \%, \mathrm{SC}_{e c}$ with $2.8 \%$ and $\mathrm{KC}$ with 
$6.2 \%$, cf. Fig. 6-b. Furthermore, our EC approach led to stable results over varying source sizes (Fig. 6-e). However, for 2 queries having large sources in their top-100 results, we observed misleading a clustering of sampled entities. Such associated clusters, in turn, led to a bad contextualization, see Fig. 6-e.

Notice that all result reorderings, either by EC or by SC, yielded an improvement (up to $6.2 \%$ ) over the plain KC baseline, cf. Fig. 6-b/e. These findings confirm results reported in [2] and clearly show the potential of data source contextualization techniques. In other words, such observations demonstrate the usefulness of contextualization - participants wanted to have additional/contextualized sources, as provided by EC or SC.

In Fig. 6-c we show the \# sources returned for contextualization of the "original" top-10 sources (obtained via $\mathrm{KC}$ ). EC returned 19\% less contextualization sources than $\mathrm{SC}_{s c}$ and $28 \%$ less than $\mathrm{SC}_{e c}$. Unfortunately, as contextualization is a "fuzzy" criteria, we could not determine the total number of relevant sources to be discovered in the top-100. Thus, no recall factor can be computed. However, combined with relevance scores in Fig. 6-b, \# sources gives a precision-like indicator. That is, we can compute the average relevance gain per contextualization source: $1.13 \mathrm{EC}, 0.89 \mathrm{SC}_{s c}$, and $0.77 \mathrm{SC}_{s c}$, see Fig. 6-b/c. Again, we explain the good "precision" of EC with the fine-grained clustering, providing better and more accurate data source semantics.

Last, it is important to note that, while Exp. 1 (Sect. 6.1) and Exp. 2 (Sect. 6.2) differ greatly in terms of their setting, our observations were still similar: EC outperformed both baselines due to its fine-grained entity clusters.

\section{Related Work}

Closest to our approach is work on contextual Web tables [2]. However, [2] focuses on flat entities in tables, i.e., entities adhere to a simple and fixed relational structure. In contrast, we consider entities to be subgraphs contained in data sources. Further, we do not require any kind of "external" information. Most notably, we do not use top-level schemata.

Another line of work is concerned with query processing over distributed RDF data, e.g., $[6,9,10,13,18]$. During source selection, these approaches frequently exploit indexes, link-traversal, or source meta-data, for mapping queries/query fragments to sources. Our approach is complementary, as it enables systems to involve users during source selection. We outlined such an extension of the traditional search process as well as its benefits throughout the paper.

Last, data integration for Web (data) search has received much attention [4]. In fact, some works aim at recommendations for sources to be integrated, e.g., [3, $14,17]$. Here, the goal is to give recommendations to identify (integrate) identical entities and schema elements across different data sources.

In contrast, we target a "fuzzy" form of integration, i.e., we do not give exact mappings of entities or schema elements, but merely measure whether or not sources contain entities that might be "somehow" related. In other words, our contextualization score indicates whether sources might refer to similar entities and may provide contextual information. Furthermore, our approach does not require data sources to adhere to a known schema - instead, we exploit data mining strategies on instance data (entities). 


\section{Conclusion}

We presented a novel approach for Web data source contextualization. For this, we adapted well-known techniques from the field of data mining. More precisely, we provide a framework for source contextualization, to be instantiated in an application-specific manner. By means of a real-world use-case and prototype, we show how source contextualization allows for user involvement during source selection. We empirically validated our approach via two user studies.

\section{References}

1. R. Chitta, R. Jin, T. C. Havens, and A. K. Jain. Approximate kernel k-means: solution to large scale kernel clustering. In SIGKDD, 2011.

2. A. Das Sarma, L. Fang, N. Gupta, A. Halevy, H. Lee, F. Wu, R. Xin, and C. Yu. Finding related tables. In SIGMOD, 2012.

3. H. R. de Oliveira, A. T. Tavares, and B. F. Lóscio. Feedback-based Data Set Recommendation for Building Linked Data Applications. In I-SEMANTICS, 2012.

4. A. Doan, A. Y. Halevy, and Z. G. Ives. Principles of Data Integration. Morgan Kaufmann, 2012.

5. S. Fausser and F. Schwenker. Clustering large datasets with kernel methods. In $I C P R, 2012$.

6. O. Görlitz and S. Staab. SPLENDID: SPARQL Endpoint Federation Exploiting VOID Descriptions. In COLD Workshop, 2011.

7. G. A. Grimnes, P. Edwards, and A. Preece. Instance based clustering of semantic web resources. In $E S W C, 2008$.

8. P. Haase, M. Schmidt, and A. Schwarte. The Information Workbench as a SelfService Platform for Linked Data Applications. In COLD Workshop, 2011.

9. A. Harth, K. Hose, M. Karnstedt, A. Polleres, K. Sattler, and J. Umbrich. Data summaries for on-demand queries over linked data. In $W W W, 2010$.

10. O. Hartig, C. Bizer, and J. Freytag. Executing SPARQL Queries over the Web of Linked Data. In $I S W C, 2009$.

11. A. K. Jain, M. N. Murty, and P. J. Flynn. Data clustering: a review. $A C M$ Computing Surveys, 1999.

12. M. Kendall and J. D. Gibbons. Rank Correlation Methods. 1990.

13. G. Ladwig and T. Tran. Linked Data Query Processing Strategies. In $I S W C, 2010$.

14. L. A. P. P. Leme, G. R. Lopes, B. P. Nunes, M. A. Casanova, and S. Dietze. Identifying Candidate Datasets for Data Interlinking. In ICWE, 2013.

15. H. Lodhi, C. Saunders, J. Shawe-Taylor, N. Cristianini, and C. Watkins. Text classification using string kernels. J. Mach. Learn. Res., 2002.

16. Lösch et al. Graph kernels for RDF data. In $E S W C, 2012$.

17. A. Nikolov and M. d'Aquin. Identifying Relevant Sources for Data Linking using a Semantic Web Index. In LDOW Workshop, 2011.

18. A. Nikolov, A. Schwarte, and C. Hütter. FedSearch: Efficiently Combining Structured Queries and Full-Text Search in a SPARQL Federation. In ISWC. 2013.

19. J. Shawe-Taylor and N. Cristianini. Kernel Methods for Pattern Analysis. 2004.

20. A. Wagner, P. Haase, A. Rettinger, and H. Lamm. Discovering related data sources in data-portals. In First International Workshop on Semantic Statistics, 2013.

21. A. Wagner, P. Haase, A. Rettinger, and H. Lamm. Entity-based Data Source Contextualization for Searching the Web of Data. Techreport, 2013. http://www . aifb.kit.edu/web/Techreport3043.

22. R. Zhang and A. Rudnicky. A large scale clustering scheme for kernel K-Means. In Pattern Recognition, 2002. 